\title{
The Anatomic Structure of Pulmonary Arteries as a Source of Unreliability in Thermodilution Cardiac Output Measurement
}

\author{
M. Gawlikowski ${ }^{a, *}$, T. Pustelny ${ }^{a}$, \\ B. Przywara-Chowaniec ${ }^{b}$ And P. Struk ${ }^{a}$ \\ ${ }^{a}$ Division of Mathematics and Physics, Silesian Technical University \\ Krzywoustego 2, 44-100 Gliwice, Poland \\ ${ }^{b}$ II Clinic of Cardiology, Silesian Medical University \\ Sklodowskiej-Curie 10, 41-800 Zabrze, Poland
}

One of the most fundamental examination in intensive care medicine is cardiac output measurement, realized by pulmonary artery catherization. The thermodilution cardiac output estimation method is not resistant to various kinds of disturbances, which significantly decreases its sensitivity and specificity. The paper depicts investigations related to analysis of presumable new source of unreliability - asymmetric blood flow through pulmonary vessels. The investigations consisted of two phases: clinical examination (to obtain anatomical data) and computer modeling (to simulate the flow symmetry).

PACS numbers: 47.63.Cb, 07.05.Tp, 87.19.Uv

\section{Introduction}

The cardiac output (CO) measurement is one of the most fundamental examination in cardiovascular system diagnostic. Despite of numerous non-invasive methods existence (e.g. Fick method, ultrasound Doppler, computer tomography) the most popular in clinical practice is thermodilution cardiac output measurement method. It is realized by pulmonary artery catherization (PAC) with application of the Swan-Ganz catheter (Fig. 1). Moreover, this kind of examination allows to measure other important hemodynamic parameters, like pulmonary artery pressure (PAP), pulmonary artery wedge pressure (PAWP — which corresponds to the

*corresponding author; e-mail: maciej.gawlikowski@poczta.onet.pl 
left atrial pressure) and pulmonary vessels resistance (PVR). The examination consists in locating the Swan-Ganz catheter into the left or right pulmonary artery (LPA or RPA), from the jugular vein, through the right atrium (RA) and right ventricle $(\mathrm{RV})$. Next, the cold indicator (isotonic salt solution, $T=1 \ldots 5^{\circ} \mathrm{C}$ ) is rapidly injected to the RA by the small diameter duct located inside the catheter [1]. The indicator concentration is measured by the temperature-sensitive element mounted at the end of catheter. The temperature vs. time curve is registered (indicator dilution curve - IDC) and CO is computed from the Steward-Hamilton formula (Eq. (1)) [2]:

$$
\Phi=\frac{c_{\mathrm{i}} \rho_{\mathrm{i}}}{c_{\mathrm{b}} \rho_{\mathrm{b}}} \frac{V_{\mathrm{i}}\left(T_{\mathrm{b}}-T_{\mathrm{i}}\right)}{\int_{t_{0}}^{\infty}(-T(t)) \mathrm{d} t},
$$

where $c_{\mathrm{i}}, c_{\mathrm{b}}$ - specific heat of indicator and blood; $\rho_{\mathrm{i}}, \rho_{\mathrm{b}}$ - specific mass of indicator and blood; $V_{\mathrm{i}}$ - volume of indicator; $T_{\mathrm{b}}, T_{\mathrm{i}}$ - temperature of blood and indicator; $T(t)$ - IDC.
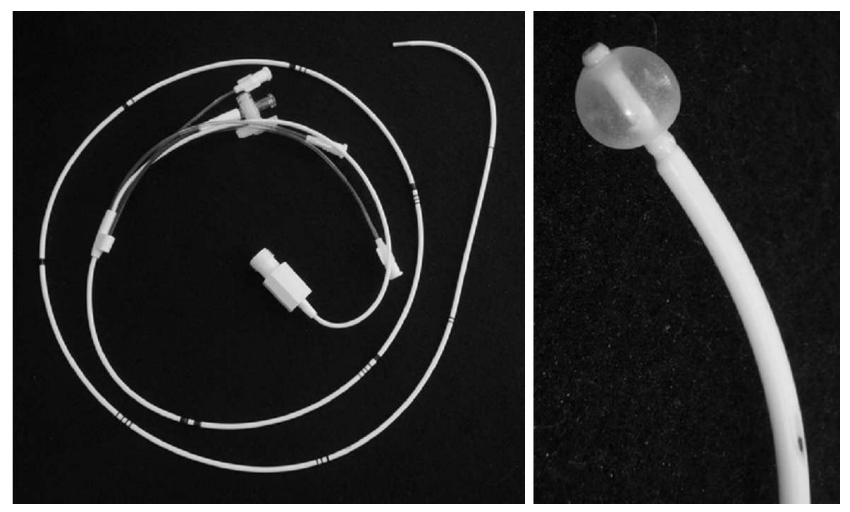

Fig. 1. The Swan-Ganz catheter (left) and pumped balloon (right).

Indeed, the PAC examination is invasive, but furnishes additional information about pressures inside the cardiovascular system and allows to its comprehensive assessment. It was proved that PAC is safe for patient and it does not increase the risk of death [3]. This examination is recommended by Polish and European Society of Cardiology [4] and it is routine in clinical practice.

\section{Goal}

The monitoring of illness progress and treatment results requires high sensitivity and specificity of applied CO measurement method. However, the PAC and CO measurement by thermodilution is acknowledged as a "gold clinical standard", but generally its accuracy is about $20 \%$. Moreover, the results of examination may be uncertain in case of accompanying diseases, like valve disease, arrhythmia or right ventricle hypertrophy. To improve the accuracy of considered method it 
is necessary to identify the sources of its unreliability - several of them were previously investigated by other authors [5].

During pulmonary artery catherization the tip of the Swan-Ganz catheter may be located in left or right pulmonary artery (Fig. 2). It is obvious that temperature sensor mounted at the catheter's tip, detects only this portion of indicator, which flows through the artery, where the sensor was located. In case, when blood flows through RPA and LPA are not the same, a different portion of indicator will flow through those arteries. In authors' opinion this effect may be another component of total method's unreliability. The goal of presented analysis was to determine if are the differences in anatomic structures of RPA and LPA influent on volumetric flow symmetry through those arteries and, in consequence, is it a systematic thermodilution CO measurement method's unreliability.

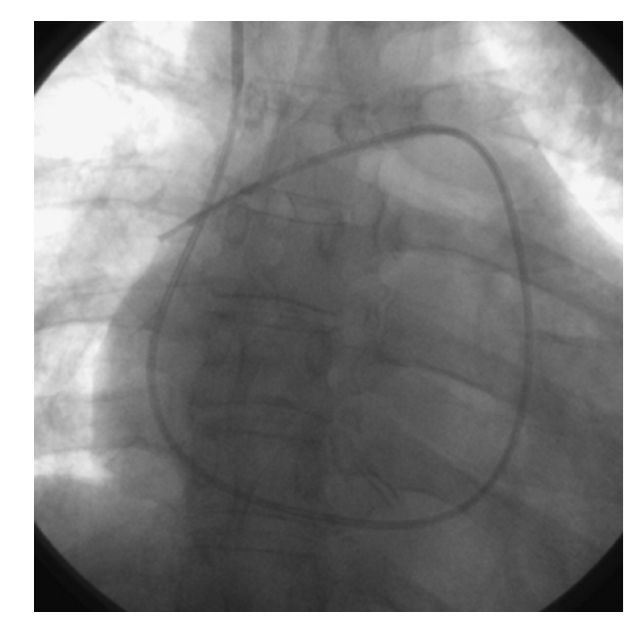

Fig. 2. X-ray of the Swan-Ganz catheter located into RPA (CO-measurement position).

\section{Clinical examinations}

The aim of clinical examinations were: to gather data for numerical model's verification and to estimate the variability of pulmonary arteries dimension.

\subsection{Material and methods (clinical)}

All clinical investigations were performed on hospitalized patients (volunteers) by ultrasound, trans-thorax heart examination. The static scans of pulmonary trunk (PT), RPA and LPA were acquired and the diameters of those anatomic structures were measured (Fig. 3). Additionally, by the Doppler method, the blood flow velocity was estimated (Fig. 4). 


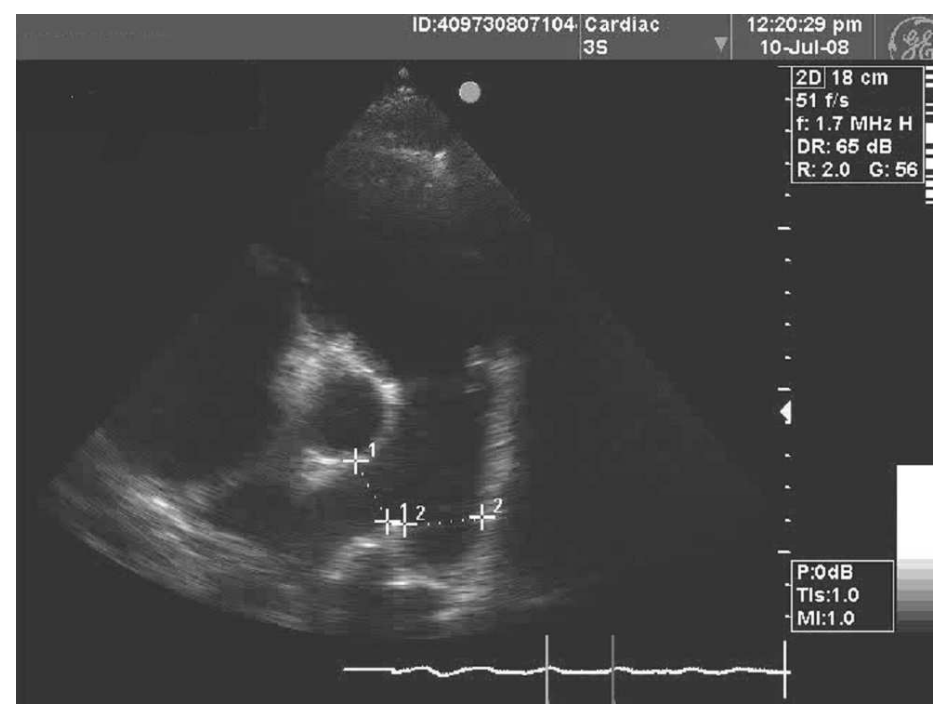

Fig. 3. The ultrasound section of pulmonary vessels - measurement of RPA (1) and LPA (2) diameters.

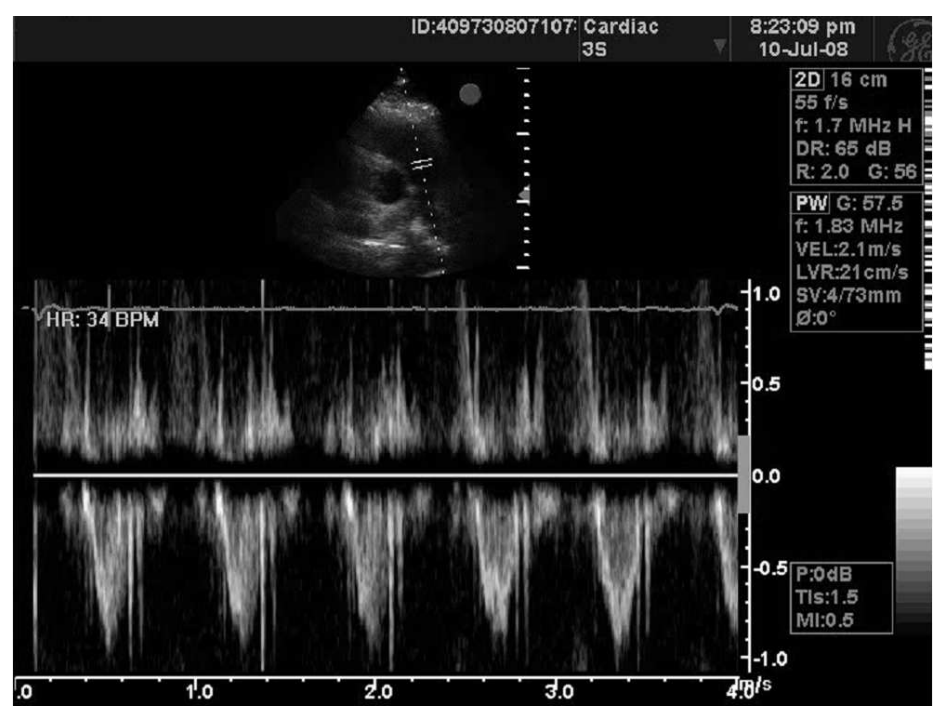

Fig. 4. The Doppler blood flow velocity measurement in PT.

\subsection{Results (clinical)}

The trans-thorax ultrasound imaging could not be performed on every patients. In several cases the obtained scans did not allow to recognize the pulmonary vessels, however the ascending aorta, left ventricle and left atrium were clearly visible. The Doppler blood flow velocity measurement was possible in PT only - the obtained flow waves in LPA and RPA were unclear. 
TABLE I

The summary of clinical examinations (M/F - male/female, HR - heart rate [beat per minute], BMI - body mass index).

\begin{tabular}{c|c|c|c|c|c}
\hline \hline & \multicolumn{2}{|c|}{$\begin{array}{c}\text { Pulmonary vessels } \\
\text { diameters }\end{array}$} & $\begin{array}{c}\text { Blood } \\
\text { velocity } \\
\text { in PT }\end{array}$ & Remarks \\
\hline $\begin{array}{c}\text { Parient } \\
\text { id. }\end{array}$ & $\begin{array}{c}\text { PT } \\
{[\mathrm{cm}]}\end{array}$ & $\begin{array}{c}\text { LPA } \\
{[\mathrm{cm}]}\end{array}$ & $\begin{array}{c}\text { RPA } \\
{[\mathrm{cm}]}\end{array}$ & $\begin{array}{c}v_{\mathrm{PT}} \\
{[\mathrm{m} / \mathrm{s}]}\end{array}$ & pulmonary hypertension \\
\hline $\begin{array}{c}2913.1 .1 .0 \\
\mathrm{M}\end{array}$ & 2.93 & 2.10 & 2.40 & $\begin{array}{c}0.63 \\
(\mathrm{HR}=85 \mathrm{BPM})\end{array}$ & \\
\hline $\begin{array}{c}2918.1 .1 .0 \\
\mathrm{M}\end{array}$ & 1.82 & 0.97 & 1.33 & $\begin{array}{c}1.2 \\
(\mathrm{HR}=100 \mathrm{BPM})\end{array}$ & - \\
\hline $\begin{array}{c}2921.1 .1 .0 \\
\text { F }\end{array}$ & 1.55 & 1.00 & 1.18 & $\begin{array}{c}0.8 \\
(\mathrm{HR}=66 \mathrm{BPM})\end{array}$ & low BMI \\
\hline
\end{tabular}

From registered ultrasound scans the following parameters were calculated: diameters of PT, LPA, RPA, maximal blood flow velocity through PT (Table I).

\subsection{Discussion (clinical)}

For all examined patients the RPA diameter is greater than LPA (experimentally obtained data were confirmed in literature $[6,7]$ ). This fact may be explained by greater blood supply to the right lung (which consists of 3 lobes) than to the left lung (which consists of 2 lobes). It should be emphasized that pulmonary circulation consists of two independent systems - the pulmonary vessels (which take part in gas exchange) and the bronchial vessels (which deliver the nutritive blood to the lungs).

The list of examination results (Table I) reveals significant anatomical variations of vessels diameter. For female the characteristic dimensions are less than for male patients. The greater vessels diameters correspond to the slower blood velocity. Moreover, it depends on heart rate (HR). The maximal blood velocity in every cases did not exceed $1.2 \mathrm{~m} / \mathrm{s}$. This data will be used to calculate the flow turbulence level in PT.

\section{Numerical modeling}

The goal of numerical modeling was to simulate the blood flow through the pulmonary vessels in order to estimate flow symmetry through the LPA and RPA. Performed numerical modeling was based on clinical data verified in literature.

\subsection{Material and methods (modeling)}

The anatomic structure of PT and its bifurcation for right and left pulmonary arteries (RPA and LPA) were reconstructed from transverse scan of human body. 


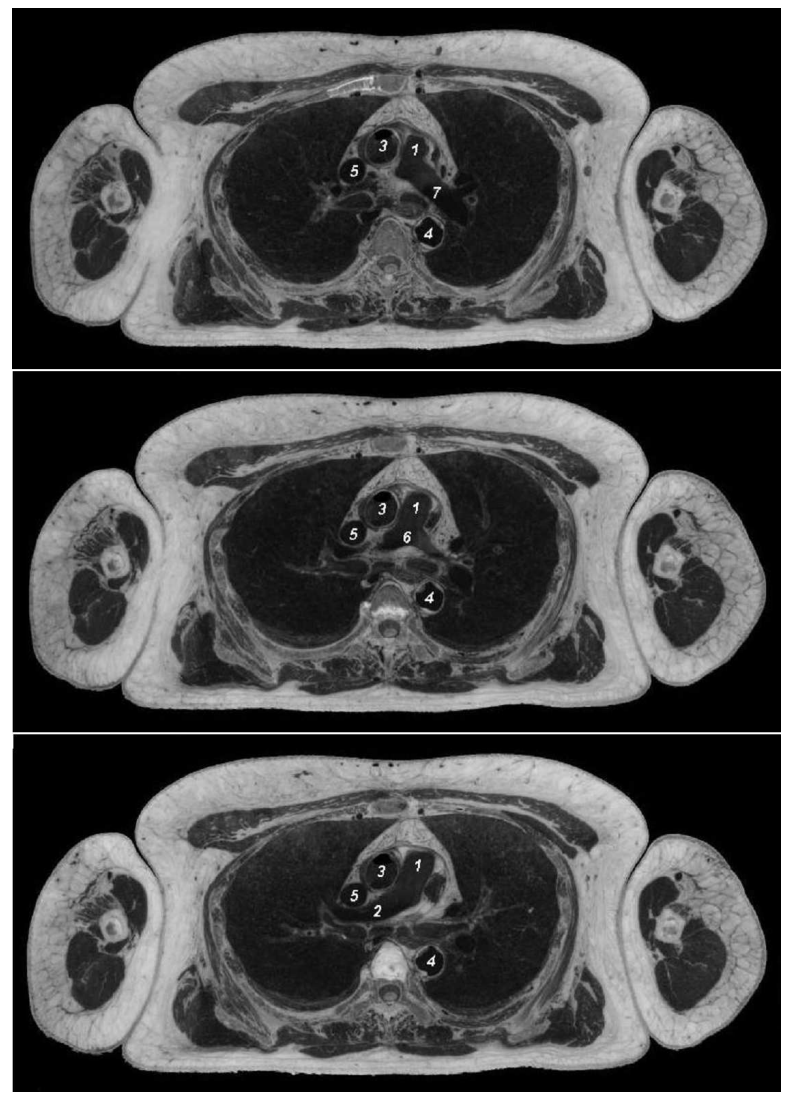

Fig. 5. Transverse section of human body (male) [8] — the pulmonary vessels were shown. 1 - pulmonary trunk (PT), 2 - right pulmonary artery (RPA), 3 - ascending aorta (AO), 4 - descending aorta (DA), 5 - vena cava (VC), 6 - pulmonary trunk bifurcation, 7 - left pulmonary artery (LPA).

The scans come from free data base of "Visible Human" project [8]. Three examples of them were depicted in Fig. 5.

The result of reconstruction was a 3D computer model of pulmonary vessels, containing information about arteries diameter, length and their orientation in three-dimensional space. In next step, the 3D numerical model was simplified (in SolidEdge CAD environment) and parameterized, to prepare it for meshing (Fig. 6). There was made an assumption that specified vessels diameter $\left(D_{\mathrm{PT}}\right.$, $\left.D_{\mathrm{RPA}}, D_{\mathrm{LPA}}\right)$ will be variable and their length will be constant. From theoretical study $[9,10]$ and clinical investigations the following vessels diameters were assumed for further numerical flow simulation: $D_{\mathrm{PT}}=20 \mathrm{~mm}, D_{\mathrm{RPA}}=16 \mathrm{~mm}$, $D_{\mathrm{LPA}}=12 \mathrm{~mm}$.

The validation of reconstructed model geometry was performed by comparison of two patterns: model's front geometry projection and ultrasonic projection 


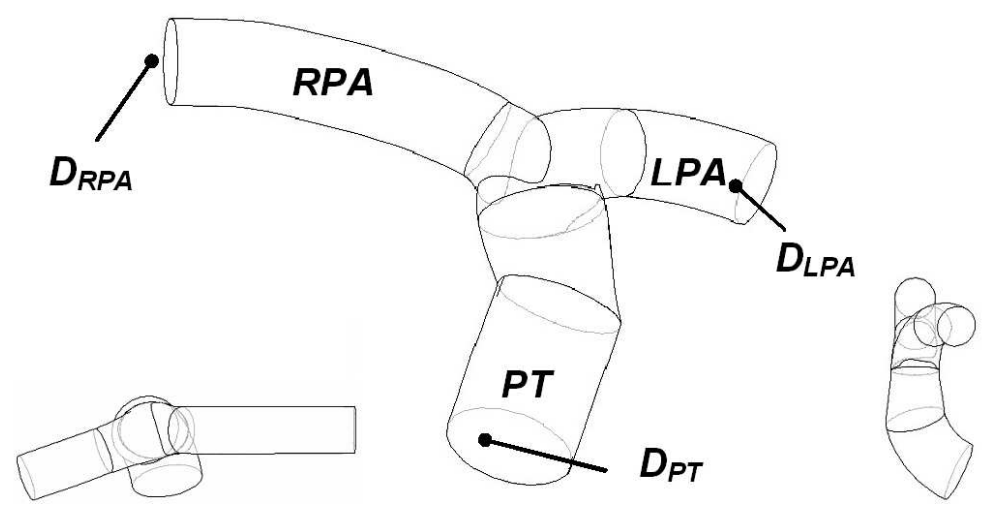

Fig. 6. The simplified and parameterized model of pulmonary vessels (upper part) and its top and side views (lower parts).

of pulmonary vessels (Fig. 7) [7]. The result of comparison turned out positive and it allowed to find a good correlation between numerical model and anatomical structures.

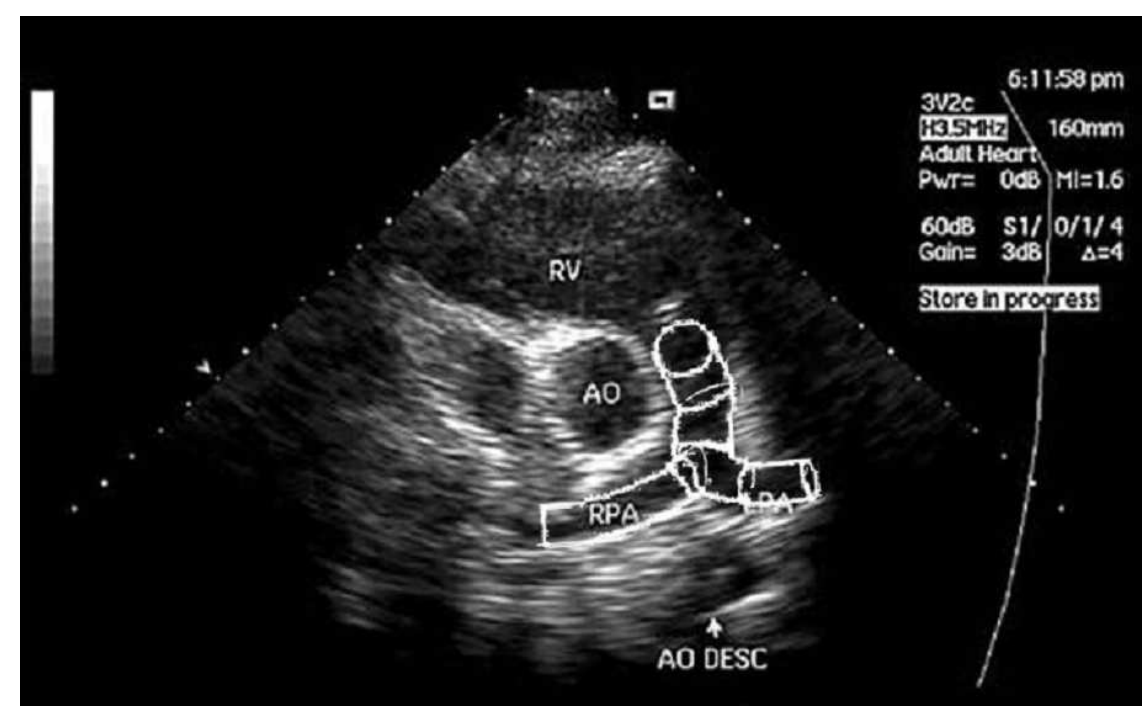

Fig. 7. Model's validation: the shape of projected pulmonary vessels (white solid line) and pulmonary vessels ultrasound projection [7].

The analyzed geometry was meshed in Gambit environment. The mesh consisted of about 78,000 tetrahedral elements and contained three faces: one velocity inlet type (at the beginning of PT) and two pressure outlet types (at the ends of LPA and RPA). The load pressures of output vessels were set to $12 \mathrm{mmHg}$, which is a typical pressure in pulmonary circulation [9]. To simulate the flow 
phenomena the finite element method (FEM) was utilized (Fluent 6.3.26 solver, single precision digits). The previous literature study and clinical non-invasive patients' examination by ultrasonic Doppler method allowed to estimate blood velocity wave in PT (Fig. 4) — its maximal value reached $1.2 \mathrm{~m} / \mathrm{s}$. By calculating the Reynolds number (Eq. (2)) the level of turbulence was estimated [11]:

$$
\operatorname{Re}=\frac{\rho_{\mathrm{B}} \nu D}{\mu} \approx 9000, \quad \operatorname{Re}>2300,
$$

where $\rho_{\mathrm{b}}$ - specific mass of blood, $v$ - velocity, $\nu$ - viscosity of blood, $D$ vessel diameter.

Indeed, for typical PT diameter $(20 \mathrm{~mm})$, the value of the Reynolds number at the blood flow's peak exceeded critical value of 2300 , but it should be assumed that it took place only at flows peaks. In other phases the turbulence level was low. It determined the applied types of viscous models: $k-\varepsilon, k-\omega$ and Reynolds's stress. The simulation were performed for all mentioned types of solvers, taking into consideration the blood viscosity and density $(\nu=0.0035 \mathrm{~kg} /(\mathrm{m} \mathrm{s}), \rho=$ $\left.1055 \mathrm{~kg} / \mathrm{m}^{3}\right)$. The blood was treated as a Newtonian liquid. The simulations were performed for continuous blood flow with velocity of $1.2 \mathrm{~m} / \mathrm{s}$.

\subsection{Results (modeling)}

The convergence of solutions were obtained for all applied solver types. The flow profiles (velocity magnitude) were depicted in Fig. 8. For various types of turbulence models the total volumetric fluxes $(\mathrm{FV})$ in all vessels were calculated
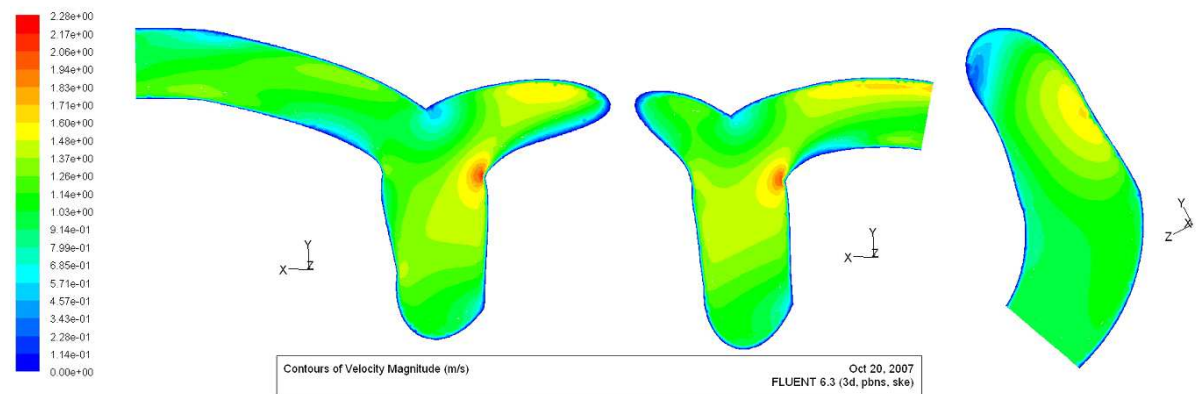

Fig. 8. The velocity flow profiles in specific vessels.

TABLE II

The RPA/LPA volumetric flow vs. various viscid models.

\begin{tabular}{c|c}
\hline \hline Viscous model & $\mathrm{FV}_{\mathrm{RPA}} / \mathrm{FV}_{\mathrm{LPA}}$ \\
\hline$k-\varepsilon$ & 1.175 \\
$k-\omega$ & 1.252 \\
Reynolds's stress & 1.169
\end{tabular}


and the $\mathrm{FV}_{\mathrm{RPA}} / \mathrm{FV}_{\mathrm{LPA}}$ were computed (refer to Table II). For all viscid models the blood flow through RPA was greater than through LPA.

\section{Conclusions}

Clinical examination revealed strongly variability of pulmonary vessels diameters, depending on individual anatomical features. Performed numerical simulations revealed asymmetric volumetric flow through the considered vessels, regardless of utilized blood viscosity model.

It is likely that the mentioned flow asymmetry is influent on cold indicator permeation in blood during thermodilution examination. This conjecture should be proved by experiments on physical model of pulmonary circulation (investigations are in progress).

\section{Acknowledgments}

This work was supported by Ministry of Science (grant No. N N518 336135) and Czesław M. Rodkiewicz Scholarship Foundation.

\section{References}

[1] J.M. Headley, Invasive Hemodynamic Monitoring: Physiological Principles and Clinical Applications, Edwards Lifescience, Irvine 2002.

[2] K.L. Zierler, Circulation Research 10, 393 (1962).

[3] D. Payen, E. Gayat, Critical Care 10 (Suppl. 3), S7 (2006).

[4] N.S. Nieminen, M. Böhm, M.R. Cowie, H. Drexler, G.S. Filippatos, G. Jondeau, Y. Hasin, J. Lopez-Sendon, A. Mebazaa, M. Metra, A. Rhodes, K. Swedberg, Polish Cardiology 63, 2 (2005) (in Polish).

[5] T. Nishikawa, S. Doshi, Canadian J. Anesthesia 40, 142 (1993).

[6] A. Bochenek, The Anatomy of Human, Vol. II, PZWL, Warsaw 1992 (in Polish).

[7] M. Darlak, M. Gawlikowski, R. Kustosz, T. Pustelny, Mol. Quant. Acoust. 27, 89 (2006).

[8] Visible human projects database - http://www.nlm.nih.gov/research/visible/.

[9] M. Krzanowski, A. Plichta, The Ultrasonic Atlas of Vessels, Practical Medicine Publ., Kraków 2000 (in Polish).

[10] A. Szczeklik, The Internal Diseases, Vol. I, The Diseases of Cardiovascular System, Practical Medicine Publ., Kraków 2005 (in Polish).

[11] M. Gawlikowski, B. Przywara-Chowaniec, T. Pustelny, P. Struk, Mol. Quant. Acoust. 28, 329 (2007). 